\title{
NOTE
}

\section{A Conducting Nanocomposite of Polystyrene with Acetylene Black}

\author{
By Pinki SAHa SARDAR, Arjun MaITY, Sanjib GHOSH, and Mukul BISWAS*
}

KEY WORDS: Polystryne / Acetylene Black / Conductivity / Nanocomposites /

During recent years increasing research attention is being paid to the preparation of conducting nanocomposites of vinyl addition polymers like polystyrene (PS), ${ }^{1}$ polypyrrole (PPY), ${ }^{2}$ polyaniline (PANI), ${ }^{3}$ polymethylmethacrylate (PMMA), ${ }^{4}$ etc. with carbon nanotube (CNT). Most of these materials are being exploited in regard to their electrical properties including electrorheology. 5

In view of the difficulty of availability of carbon nanotubes and their high cost any cheaper alternative for the CNT component is these composites would be indeed worth exploring. In this context we have successfully used one cheap and abundant allotrope of carbon namely acetylene black (AB) as the nanocomponent. The particle size of $\mathrm{AB}$ is in the nano order range and the material is highly conducting. We have reported that $N$-vinyl carbazole (NVC) monomer can be readily polymerized in bulk or in toluene in presence of $\mathrm{AB}$ without any extraneous catalyst; and a PNVC-AB nanocomposite can be isolated from the system. The composite possesses high dc conductivity and enhanced thermal stability compared to PNVC homopolymer. ${ }^{6}$

While extending the above procedures for polymerization vis-a-vis composite formation between $\mathrm{AB}$ and vinyl polymers such as PMMA, polyacrylonitrile $(\mathrm{PAN})^{7,8}$ it was noted that unlike NVC, acrylonitrile (AN) or methyl methacrylate (MMA) monomers could not be bulk polymerized by $\mathrm{AB}$ alone. We, therefore, modified the procedure in which MMA or AN monomers were dissolved in aqueous medium in which acetylene black was kept dispersed by sonication and nanocomposites of PMMA-AB and PAN-AB could be isolated.

To prepare polystyrene-acetylene black nanocomposite (PS-AB) we used the emulsion polymerization process since styrene monomer is insoluble in water. Sodium dodecyl sulphate (NaDS) was used as an emulsifier and $\mathrm{K}_{2} \mathrm{CrO}_{4}$ and $\mathrm{NaAsO}_{2}$ were used as the redox initiator. Details of these procedures and bulk property characterizations of the PS-AB nanocomposite are highlighted in this article. It may be of interest to point out here that Choi et al. ${ }^{1}$ reported the preparation of carbon nanotube (CNT) adsorbed polystyrene and PMMA microspheres by using nanotubes sonicated in aqueous solutions of the surfactants to which PS or PMMA microspheres were dropped. The conductivity values of
$2 \times 10^{-4}-3 \times 10^{-5} \mathrm{~S} / \mathrm{cm}$ were observed for CNT adsorbed on PS microspherers using cetavelon or sodium dodecyl sulphate as surfactant respectively.

\section{EXPERIMENTAL}

\section{Materials}

Styrene monomer was freed from inhibitor by washing with $5 \% \mathrm{NaOH}$ solution, followed by distilled water, and after drying over anhydrous sodium sulphate was finally fractionated. Potassium chromate (Riedel, Germany) and sodium arsenite (Riedel, Germany) were used as the oxidant and the reductant respectively. Acetylene black (SENKA India, Chennai, India) was preheated by heating at $120^{\circ} \mathrm{C}$ for $2 \mathrm{~h}$ in vacuum prior to use. All other solvents were of analytical grade and were freshly distilled before use.

Polymerization of Styrene Monomer with $\mathrm{K}_{2} \mathrm{CrO}_{4}-\mathrm{NaAsO}_{2}$ Redox System. $0.5 \mathrm{gm}$ sodium dodecyl sulphate was dissolved in $30 \mathrm{~mL}$ of distilled and deaerated water taken in a Pyrex flask for about $15 \mathrm{~min}$. To this solution $1.5 \mathrm{~mL}$ of styrene was injected. Then the system was stirred at ambient temperature under nitrogen atmosphere for about $1 \mathrm{~h}$. Thereafter $0.1 \mathrm{~g}$ of $\mathrm{K}_{2} \mathrm{CrO}_{4}$ was added and stirred for $5 \mathrm{~min}$ and $0.3 \mathrm{~g} \mathrm{NaAsO}$ was added into this solution. The polymerization reaction was continued for $3 \mathrm{~h}$. The white polymer obtained was centrifuged, washed successfully with distilled water and methanol, and finally dried under vacuum for about $12 \mathrm{~h}$.

Preparation of Polystyrene-AB (PS-AB) Composite. $0.5 \mathrm{gm}$ sodium dodecyl sulphate was dissolved in a $30 \mathrm{~mL}$ of distilled and deaerated water taken in a Pyrex flask for about $15 \mathrm{~min}$. To this solution $1.5 \mathrm{~mL}$ of styrene was injected. Then the system was stirred at ambient temperature under nitrogen atmosphere for about $1 \mathrm{~h}$. To this solution, varying amounts of $A B$ were added (Table I). The system was kept under stirring for $30 \mathrm{~min}$ to make a suspension of $\mathrm{AB}$. Thereafter $0.1 \mathrm{~g}$ of $\mathrm{K}_{2} \mathrm{CrO}_{4}$ was added and stirred for $5 \mathrm{~min}$ and $0.3 \mathrm{~g} \mathrm{NaAsO}_{2}$ was added into this solution. The polymerization reaction was continued for $3 \mathrm{~h}$. The whole mass was poured into an excess of $\mathrm{MeOH}$. The separated black mass was filtered and washed with $\mathrm{MeOH}$ to remove any unreacted monomer followed by acetone successively and dried under vacuum at $100^{\circ} \mathrm{C}$ for $12 \mathrm{~h}$ and subsequently characterized as the PS-AB nanocomposite.

\footnotetext{
Department of Chemistry, Presidency College, 86/1 College Street, Kolkata-700073, India

*To whom correspondence should be addressed (Tel: +9133-2521-3556, +919051340721, E-mail: mukulbiswas007@gmail.com, mukul_bisaws@vsnl.net).
} 
Table I. Some typical data on polymerization of styrene, polystyrene-AB composite formation ${ }^{\mathrm{a}}$ and dc conductivity values

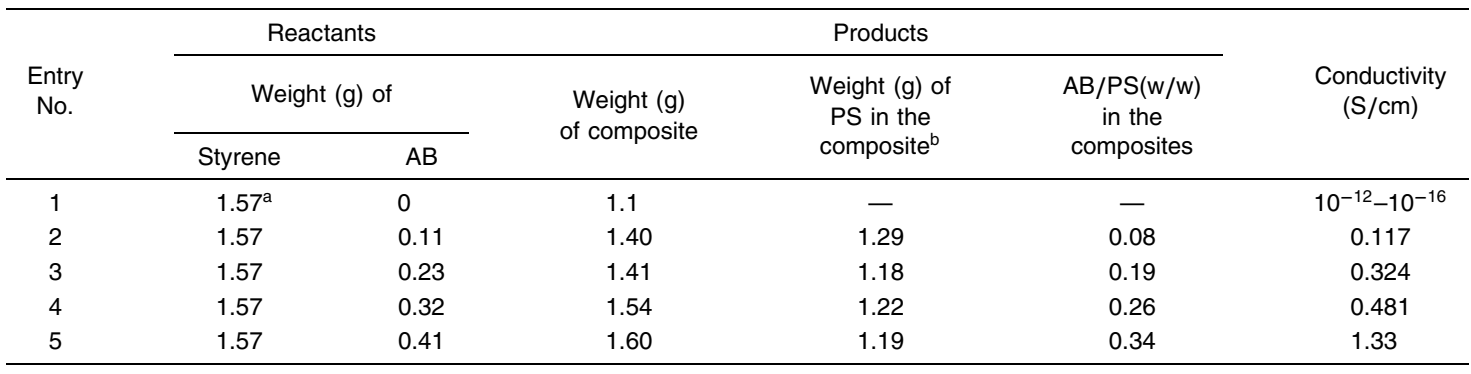

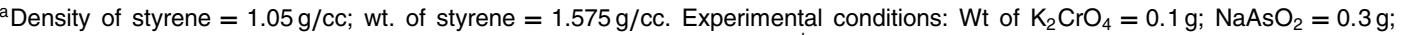

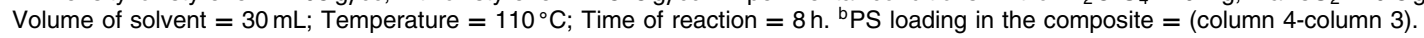

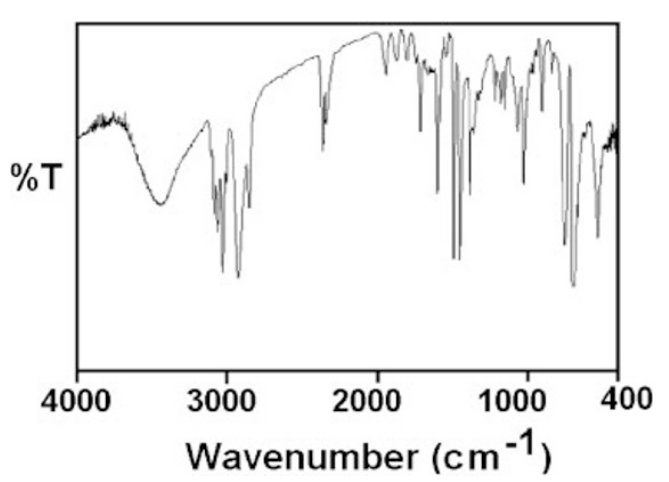

Figure 1. The FT-IR spectrum of the polymer isolated from PS-AB composite.

Characterization and Property Evaluation. The FT-IR spectrum of the mass extracted from PS-AB composite was taken on a JASCO-680 plus instrument in pressed $\mathrm{KBr}$ pellet. Dispersions of Free PS resin and PS-AB composites in 2propanol were microsprayed on a mica substrate. The samples were sputter coated with gold layer and ULTRA 55-36-11 scanning electron microscope was used to take the micrographs. Direct current (dc) resistivity measurements were conducted on pressed pellets $(13 \mathrm{~mm}$ diameter, $0.1 \mathrm{~mm}$ breadth) with silver coating, using four-probe technique. The unit manufactured by Scientific Equipment and Services, Roorke, India, comprised a low constant current source (Model LCS-02), a digital microvoltmeter (Model DMV-001) and a PID controlled oven (Model PID-200).

\section{RESULTS AND DISCUSSION}

The composite was isolated as a blackish powdery mass characteristically different from PS homopolymer which exist as a tacky mass. Table I presents some quantitative data on the polymerization vis-a-vis composite formation under the experimental condition used.

The incorporation of PS in the composite was endorsed by the FT-IR spectrum (Figure 1) of the mass recovered after refluxing the $\mathrm{PS}-\mathrm{AB}$ composite in toluene and precipitating the polymer by methanol addition matches with the same for polystyrene homopolymer. The major characteristic vibration
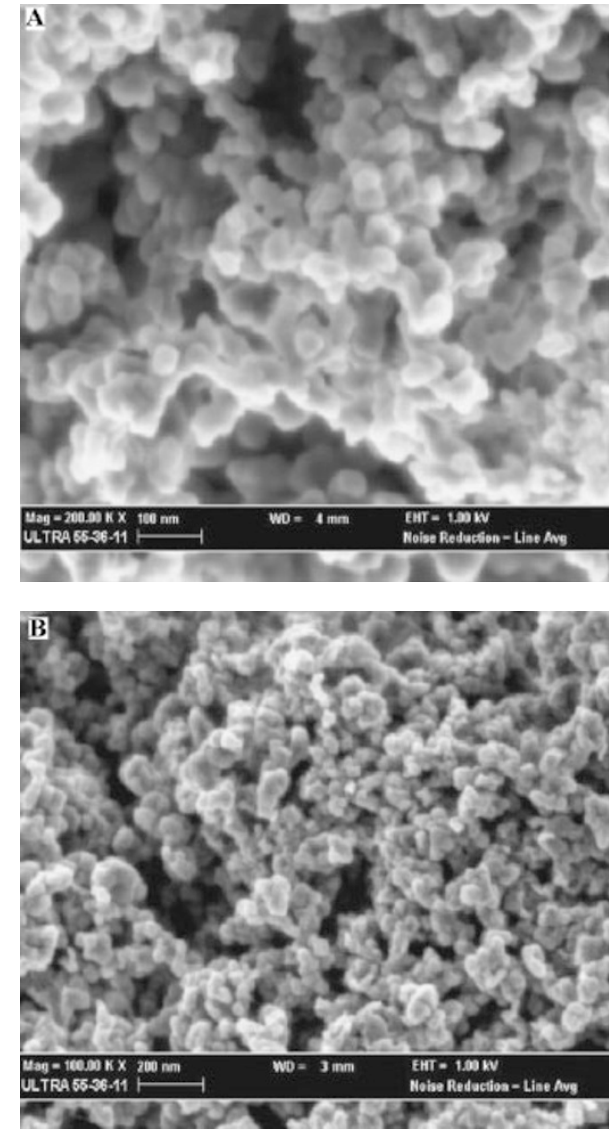

Figure 2. Scanning electron micrographs of (A) PS homopolymer, (B) PSAB composite.

bands in Figure 1 agree very convincingly with the literature data for polystyrene; ${ }^{9} \sim 3025-3060 \mathrm{~cm}^{-1}$ (aromatic C-H stretching); $2920-2850 \mathrm{~cm}^{-1}$ (Aliphatic C-H stretching); $1450-1490 \mathrm{~cm}^{-1}$ (in plane bent-stretching phenyl ring) and $700 \mathrm{~cm}^{-1}$ (out of plane phenyl ring); $750 \mathrm{~cm}^{-1}$ (out of plane hydrogen). The broad absorption around $3500 \mathrm{~cm}^{-1}$ is due to some contamination. (alcohol)

Scanning electron micrographs of PS (Figure 2A) show existence of spherical particles with average sizes in $50-70 \mathrm{~nm}$ range calculated over a wide cross-section of the micrograph. The corresponding SEM for PS-AB (Figure 2B) shows the 


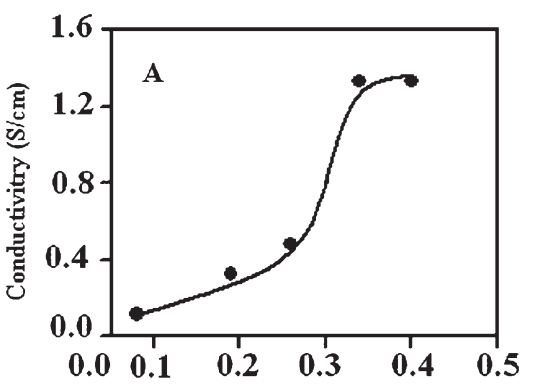

$\mathrm{AB} / \mathrm{PS}(\mathrm{w} / \mathrm{w})$ in the composites
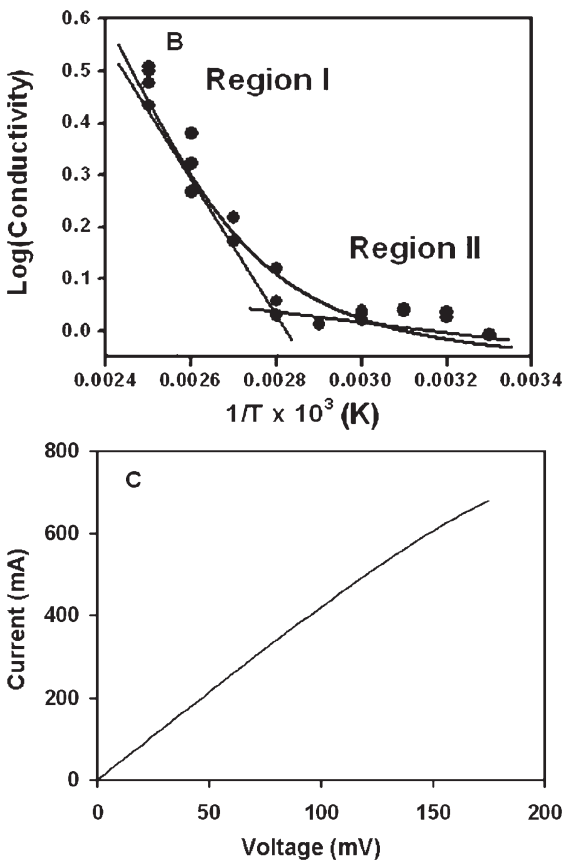

Figure 3. Conductivity characteristics of (A) PS-AB composite as a function of $A B$ loading, (B) Log (Conductivity)-1/T (Kelvin) curve of the PS$\mathrm{AB}$ composite, (C) Current-voltage (I-V) curve for PS-AB composite.

formation of discrete aggregates of particles with sizes in the $140-160 \mathrm{~nm}$ range. Evidently in the composite AB nanoparticles must have been cemented together by the PS moieties during polymerization. This is reflected in the larger aggregate sizes manifesting in the composites.

Figure $3 \mathrm{~A}$ shows that the room temperature conductivity of the PS-AB composites increases initially with increasing $A B$ : $\mathrm{PS}(\mathrm{w} / \mathrm{w})$ ratio in the composite and tends to level off with $\mathrm{AB}$ amounts 0.4 to 0.5 in the initial feed under the conditions followed in the experiments. Since PS is highly non-conducting the conductivity of pure $\mathrm{AB}(3.7 \mathrm{~S} / \mathrm{cm})$ will never be realized in the composite. Log (conductivity) vs. 1/T plot (Figure 3B) for PS-AB (entry 4 in Table I) shows a change of slope implying presence of intrinsic conduction (region I) and extrinsic conduction (region II). ${ }^{10}$ The latter is usually observed in semiconductor systems due to impurity defects. ${ }^{11}$ The activation energy of intrinsic conduction as calculated from the slope in region I is estimated to be $0.008 \mathrm{ev} / \mathrm{mol}$. Relevantly we recently realized ${ }^{12}$ a similar activation energy value for a polyacrylamide- $\mathrm{AB}$ nanocomposite. For graphite, literature suggests a value of $0.005 \mathrm{ev} / \mathrm{mol} .{ }^{11}$ Hence the value realized in $\mathrm{AB}$ based nanocomposites of PS or PAA systems appears to be reasonable. As observed with other $\mathrm{AB}$ based systems and also metal oxides/13X-zeolite based systems of PPY, PANI, PF $^{8}$ and $\mathrm{PNVC}^{6,13}$ the current-voltage variation (Figure 3C) in PS$\mathrm{AB}$ composite is linear implying metallic behaviour. ${ }^{10}$ It may be relevant to add here that the conductivity of the PS-AB composites prepared in this work is significantly higher than the CNT-PS composites reported by Choi et al. ${ }^{1}$

Acknowledgment. The authors thank the Department of Science \& Technology, Govt. of India, New Delhi, India, for funding a project (No. SR/S5/NM-14/2003) to MB and a SRF position to PSS and the authorities of Presidency College for facilities.

Received: December 18, 2008 Accepted: May 7, 2009 Published: July 9, 2009

\section{REFERENCES}

1. H.-J. Jin, H. J. Choi, S. H. Yoon, S. J. Myung, and S. E. Shin, Chem. Mater., 17, 4034 (2005).

2. K. H. An, K. K. Jeon, J. K. Heo, S. C. Lim, D. J. Bac, and Y. H. Lee, J. Electrochem. Soc., 149, A1058 (2002).

3. W. K. Maser, A. M. Benito, M. A. Callejas, T. Seeger, M. T. Martinez, J. Schreiber, J. Muszynski, O. Chauvet, Z. Osvath, A. A. Koos, and L. P. Biro, Mater. Sci. Eng., C, 23, 87 (2003).

4. a) J. H. Sung, H. S. Kim, H.-J. Jin, H. J. Choi, and I.-J. Chin, Macromolecules, 37, 9899 (2004).

b) S. J. Park, M. S. Cho, H. J. Choi, and M. S. Jhon, Macromol. Rapid Commun., 24, 1070 (2003).

c) M. Biswas and S. S. Roy, Adv. Polym. Sci., 155, 167 (2001).

5. A. Maity and M. Biswas, J. Ind. Eng. Chem., 12, 311 (2006).

6. N. Ballav, A. Maity, and M. Biswas, Mater. Chem. Phys., 87, 120 (2004).

7. A. Maity and M. Biswas, Int. J. Polym. Mater., 55, 175 (2006).

8. A. Maity and M. Biswas, Polym. J., 36, 812 (2004).

9. R. L. Wintermyer and W. C. Buzanowski, in "Concise Encyclopedia of Polymer Science and Engineering," J. I. Kroschwitz, Ed., John Wiley and Sons, New York, 1990, p 1129.

10. J. Wang, J. Dai, and T. Yarlagadda, Langmuir, 21, 9 (2005).

11. a) A. Tager, "Physical Chemistry of Polymers," Mir Publishing, Moscow, 1972, pp. 303-310.

b) H. Meier, in "Dark and Photoconductivity of Organic Solids, Verlag Chemie," 1974, Chapter 3, p 39.

12. P. K. Verma, P. Saha Sardar, S. Ghosh, and M. Biswas, Polym. Compos., 30, 490 (2009).

13. D. Ghosh and M. Biswas, J. Polym. Res., 16, 245 (2009). 